E D I T O R I A L S

\title{
Prophylaxis and Aspergillosis - Has the Principle Been Proven?
}

\author{
Ben E. De Pauw, M.D., and J. Peter Donnelly, Ph.D.
}

Invasive fungal diseases contribute substantially to death and illness associated with the prolonged, profound neutropenia resulting from intensive chemotherapy for hematologic cancers and from myeloablation for allogeneic hematopoietic stemcell transplantation. In addition, the development of graft-versus-host disease (GVHD) puts transplant recipients at risk for infection for several weeks or months after engraftment. ${ }^{1}$ Given the high mortality associated with invasive fungal diseases and our inability to recognize active cases reliably, it is not surprising that prophylaxis is perceived as a sensible therapeutic approach.

More than a decade ago, fluconazole was shown to be effective for preventing candida infection. ${ }^{2}$ However, aspergillus species and other molds are not affected by this drug. Itraconazole has a spectrum of activity that includes aspergillus species, but its use is limited by its erratic uptake in the body. Although the bioavailability of itraconazole is substantially improved with an oral solution, compliance and the propensity of the drug to interact with other agents are not. ${ }^{3}$ The pathophysiology of invasive aspergillosis differs fundamentally from that of invasive candidiasis. Colonization of the mucosal membranes by candida precedes invasive disease, whereas aspergillus and other molds are contracted through the respiratory tract. Since there appears to be a positive correlation between efficacy and adequate serum levels of itraconazole, it is tempting to assume that the antiaspergillus effect will be exerted primarily through systemic antifungal activity. ${ }^{4}$

The arrival of the new azole drug, posaconazole, was most welcome, since it not only is active in vitro against a wide range of yeasts (including candida species) and molds (including aspergil- lus species, Zygomycetes, and fusarium species) but also appears to have an acceptable adverseevent profile and is well tolerated. In this issue of the Journal, two controlled trials that were designed to evaluate the prophylactic use of posaconazole are reported..$^{5,6}$

One study, by Cornely et al., was a prospective, randomized, trial involving evaluators who were unaware of the treatment assignments and patients who were undergoing chemotherapy for acute leukemia or myelodysplastic syndromes. Posaconazole was compared with either fluconazole or itraconazole for the prevention of invasive fungal disease. ${ }^{5}$ Prophylaxis was started with the first cycle of chemotherapy and was continued until recovery from neutropenia and complete remission, until the occurrence of an invasive fungal disease, or for up to 12 weeks, whichever came first. The incidences of proven and probable invasive fungal diseases were significantly lower in the posaconazole group (2\%) than in the fluconazole or itraconazole group $(8 \%)$. There were also fewer cases of invasive aspergillosis after posaconazole prophylaxis (1\%) than after fluconazole or itraconazole prophylaxis (7\%).

The other study, by Ullmann et al., was a randomized, double-blind trial of oral posaconazole or oral fluconazole as prophylaxis against invasive fungal disease in hematopoietic stem-cell transplant recipients who were also receiving immunosuppressive therapy for GVHD. ${ }^{6}$ The primary end point was the incidence of proven or probable invasive fungal disease within 16 weeks after randomization. Posaconazole and fluconazole appeared to be equally efficacious in preventing all invasive fungal diseases (incidence, 5.3\% and $9.0 \%$, respectively), but posaconazole pre- 
vented more cases of proven or probable invasive aspergillosis $(2.3 \%)$ than did fluconazole $(7.0 \%)$. There were fewer deaths in the posaconazole group, and the drug was well tolerated and relatively safe.

Both studies aimed to address efficacy: Does posaconazole work as prophylaxis in these two groups of patients? The answer is yes. One may quibble about the details of the studies. For instance, why is it that the GVHD study by Ullmann et al. was double-blind and placebo-controlled but the study of neutropenia by Cornely et al. was not? There is also the curious observation by Cornely et al. that most cases of probable aspergillosis were based on a positive test for aspergillus galactomannan antigen, not necessarily a positive culture or compatible histopathological findings. Remove these probable cases, and the advantage for posaconazole vanishes. It would be useful to know more about these cases and also whether there were similar cases for which the galactomannan test was negative in the posaconazole group, since concentrations of the antigen are affected by antifungal drugs that are active against mold. ${ }^{7}$ Although this was not stated explicitly, we assume that patients who were unable to take oral medication were excluded from the GVHD trial by Ullmann et al., because posaconazole can be given only orally. If this was indeed true, there may have been an unintentional selection of lesssick patients, which might explain the rather low incidence of invasive aspergillosis in the fluconazole group. Neither study reported whether the ability to eat a fatty diet, to help drug absorption, or compliance was a prerequisite for inclusion, and the serum concentrations of the study drug were not noted. This information is important, since one wonders whether clinical success was achieved mainly in patients with adequate levels of the drug.

How generalizable are these results, and how effective will a strategy of posaconazole prophylaxis actually prove to be? In our center, we do not use primary prophylaxis at all for patients with GVHD or with prolonged neutropenia, preferring to rely on an integrated-care plan that involves twice-weekly screening for galactomannan and a low threshold for ordering computed tomography of the chest to detect abnormal pulmonary signs, exactly as reported by Maertens et al. ${ }^{8}$ Only patients with pulmonary abnormalities consistent with those due to invasive fungal disease, or probably due to such disease, are treated.
We use this treatment strategy because the overall incidence of invasive aspergillosis in our center is low, about $4.5 \%$. Were we to use posaconazole therapy, the number of patients who would need to be treated to prevent one fungal infection (the number needed to treat) would be approximately 22, not 16 as reported by Cornely et al. There is the rub. McQuay and Moore ${ }^{9}$ posed four questions that need to be considered before adopting prophylaxis. First, would the event we are trying to prevent (i.e., invasive fungal disease) be difficult to treat if it occurred? Given the availability of voriconazole and liposomal amphoteri$\operatorname{cin} \mathrm{B}$, the answer would be no, provided we detected the invasive fungal disease sufficiently early. Second, is invasive fungal disease a serious event? The answer is a clear yes. Third, is prophylaxis safe and tolerated well enough (i.e., are there few adverse effects)? The results of the studies by Ullmann et al. and Cornely et al. suggest that the answer is yes. Fourth, is the prophylaxis effective (i.e., is the number needed to treat relatively low, such as $<20$ )? The answer for our center is no. However, for a number needed to treat of 16, as in the study of neutropenia by Cornely et al., the answer would be yes - so the decision in favor of prophylaxis hinges on the first question about how difficult the event would be to treat. The answer would be different for centers vigilantly pursuing a preemptive approach and for centers that do not have the facilities or the means to do so. Centers treating patients with GVHD are not relieved from the dilemma either: Should the centers offer prophylaxis to approximately 27 at-risk patients in order to prevent the development of invasive aspergillosis in 1 , or should no action be taken until invasive aspergillosis is suspected? Whether to use prophylaxis at all remains a choice that is pragmatic and depends on factors other than the drug in question.

The scientific community now has the results of two phase 3 studies of posaconazole prophylaxis to ponder, without the drug having first won recognition as the primary therapy for invasive aspergillosis. With posaconazole, the management of aspergillosis among high-risk patients has only become more puzzling: posaconazole appears to be the drug of choice for prophylaxis, whereas voriconazole remains the preferred treatment for proven or probable aspergillosis, ${ }^{10}$ leaving caspofungin and liposomal amphotericin B as the options for empirical therapy. ${ }^{11,12}$ This is the unfortunate consequence of mixing efficacy 
studies that should explore the options and limitations of a drug with strategic trials that, ideally, should assess the appropriate moment to administer a drug with an established efficacy.

Dr. De Pauw reports receiving consulting or lecture fees from Basilea Pharmaceuticals, Gilead Sciences, Merck, Pfizer, ScheringPlough, and Vicuron Pharma. Dr. Donnelly reports receiving consulting or lecture fees from Janssen Pharmaceuticals, Gilead Sciences, and Pfizer and grant support from AM-Pharma BV and Schering-Plough. No other potential conflict of interest relevant to this article was reported.

From the Department of Blood Transfusion and Transplant Immunology and the Department of Hematology, University Medical Center St. Radboud, Nijmegen, the Netherlands.

1. Wald A, Leisenring W, van Burik JA, Bowden RA. Epidemiology of Aspergillus infections in a large cohort of patients undergoing bone marrow transplantation. J Infect Dis 1997;175:145966.

2. Goodman JL, Winston DJ, Greenfield RA, et al. A controlled trial of fluconazole to prevent fungal infections in patients undergoing bone marrow transplantation. N Engl J Med 1992;326:84551.

3. Marr KA, Crippa F, Leisenring W, et al. Itraconazole versus fluconazole for prevention of fungal infections in patients receiving allogeneic stem cell transplants. Blood 2004;103:1527-33.

4. Glasmacher A, Prentice A, Gorschluter M, et al. Itraconazole prevents invasive fungal infections in neutropenic patients treated for hematologic malignancies: evidence from a meta-analysis of 3,597 patients. J Clin Oncol 2003;21:4615-26.

5. Cornely OA, Maertens J, Winston DJ, et al. Posaconazole vs. fluconazole or itraconazole prophylaxis in patients with neutropenia. N Engl J Med 2007;356:348-59.

6. Ullmann AJ, Lipton JH, Vesole DH, et al. Posaconazole or fluconazole for prophylaxis in severe graft-versus-host disease. N Engl J Med 2007;356:335-47.

7. Marr KA, Laverdiere M, Gugel A, Leisenring W. Antifungal therapy decreases sensitivity of the Aspergillus galactomannan enzyme immunoassay. Clin Infect Dis 2005;40:1762-9.

8. Maertens J, Theunissen K, Verhoef G, et al. Galactomannan and computed tomography-based preemptive antifungal therapy in neutropenic patients at high risk for invasive fungal infection: a prospective feasibility study. Clin Infect Dis 2005;41:1242-50. 9. McQuay HJ, Moore RA. Using numerical results from systematic reviews in clinical practice. Ann Intern Med 1997;126:712-20. 10. Herbrecht R, Denning DW, Patterson TF, et al. Voriconazole versus amphotericin $\mathrm{B}$ for primary therapy of invasive aspergillosis. N Engl J Med 2002;347:408-15.

11. Walsh TJ, Finberg RW, Arndt C, et al. Liposomal amphotericin B for empirical therapy in patients with persistent fever and neutropenia. N Engl J Med 1999;340:764-71.

12. Walsh TJ, Teppler H, Donowitz GR, et al. Caspofungin versus liposomal amphotericin B for empirical antifungal therapy in patients with persistent fever and neutropenia. N Engl J Med 2004;351:1391-402.

Copyright @ 2007 Massachusetts Medical Society.

\title{
Treatment of Uterine Fibroids — Is Surgery Obsolete?
}

\author{
Togas Tulandi, M.D., M.H.C.M.
}

In the early 1990s, Jacques H. Ravina first applied the technique of embolization of uterine arteries to treat uterine fibroids in women at high risk for complications during surgery ${ }^{1}$ in an effort to control uterine bleeding. Embolization was then expanded for the treatment of patients who were undergoing myomectomy in order to decrease intraoperative bleeding. In 1993, Ravina and colleagues started using uterine-artery embolization as a primary treatment for uterine fibroids.

Today, interventional radiologists worldwide perform uterine-artery embolization. Most of them embolize the uterine arteries bilaterally and not only the branch supplying blood to a particular fibroid (Fig. 1). In observational studies, embolization has been followed by a significant reduction in uterine volume, a decrease in excessive uterine bleeding, a low rate of subsequent hysterectomy, and a high rate of sustained symptom control (up to $80 \%$ ) 5 years after the procedure..$^{2-4}$ However, comparisons of uterine-artery embolization with other treatments on the basis of observational data are limited by the inherent dif- ferences in women who are referred for one treatment instead of another.

In this issue of the Journal, Moss et al., writing for the Randomized Trial of Embolization versus Surgical Treatment for Fibroids (REST) Investigators, ${ }^{5}$ report on the results of a randomized, multicenter trial comparing uterine-artery embolization with abdominal surgery in women with symptomatic uterine fibroids. The investigators randomly assigned 106 women to undergo embolization and 51 to undergo surgery (including 43 hysterectomies and 8 myomectomies). On the basis of results on the Medical Outcomes Study 36-Item Short-Form General Health Survey questionnaire, they found no significant differences in the quality-of-life scores between the two groups at 1 year, although symptom scores were better in the surgical group at that follow-up assessment. Complication rates were similar at 1 year in the two groups, although the study was not powered to detect differences in these rates or to detect rare complications. Of note, complications generally occurred earlier in the surgical group (typi- 